\title{
COVID-19 Detection from Chest X-Ray Images Using CNN Models: Further Evidence from Deep Transfer Learning
}

\author{
Mohamed Samir Boudrioua ${ }^{1 *}$ \\ ${ }^{1}$ Ronin Institute, Montclair, NJ, USA \\ mohamed.samir.boudrioua@ronininstitute.org
}

Recommended Citation: Boudrioua, MS. COVID-19 detection from chest X-ray images using CNN models: Further evidence from deep transfer learning. Univ Louisville J Respir Infect 2020; 4(1):Article 53. doi: 10.18297/jri/vol4/iss1/53.

\begin{abstract}
Introduction: The early automatic diagnosis of the novel coronavirus (COVID-19) disease could be very helpful to reduce its spread around the world. In this study, we revisit the identification of COVID-19 from chest X-ray images using deep learning.
\end{abstract}

Methods: We collected a relatively large COVID-19 dataset-compared with previous studies-containing 309 real COVID-19 chest X-ray images. We also prepared 2,000 chest X-ray images of pneumonia cases and 1,000 images of healthy controls. Deep transfer learning was used to detect abnormalities in our image dataset. We fine-tuned three, pre-trained convolutional neural network (CNN) models on a

\section{Introduction}

On December 2019, the novel coronavirus (COVID-19) has started to spread in China, then in other multiple countries around the world.[1-3] The early automatic diagnosis of this disease may be very beneficial for reducing its spread.[4] Deep Learning is one artificial intelligence method that can be helpful in detecting COVID-19 infections from medical images such as $X$-ray images, specifically when we have a small image dataset.[2, 4,5]

Earlier studies have used Deep Learning for the detection of COVID-19 from chest X-ray images. Minaee $e t$ al. prepared a dataset of 5,071 chest X-ray images including 71 COVID-19 images and 5000 non COVID-19 images.[2] They selected 40 COVID-19 images and 3,000 non COVID-19 to include in the test set, as well as 31 COVID-19 (496 after augmentation) and 2,000 non COVID-19 images for the training set. They trained four popular Deep Learning models, including ResNet18, ResNet50, DenseNet-121, and SqueezeNet, to detect COVID-19 infections. The best performing training dataset: DenseNet 121, NASNetLarge, and NASNetMobile.

Results: The evaluation of our models on a test dataset showed that these models achieved an average sensitivity rate of approximately $99.45 \%$ and an average specificity rate of approximately $99.5 \%$.

Conclusion: A larger dataset of COVID-19 X-ray images could lead to more accurate and reliable identification of COVID-19 infections using deep transfer learning. However, the clinical diagnosis of COVID-19 disease is always necessary.

model achieved a sensitivity rate of $97.5 \%$, and $95 \%$ of specificity.[2] Apostolopoulos and Mpesiana evaluated the performance of different state-of-the-art CNNs architectures on two datasets.[4] The first one contains 1427 chest X-ray images including 224 images with COVID-19 infection, 700 images with confirmed common bacterial pneumonia, and 504 images of healthy cases. The second dataset includes 224 images with COVID-19 infection, 714 images with confirmed bacterial and viral pneumonia, as well as 504 images of healthy cases.[4] According to their results, the best achieved accuracy, sensitivity, and specificity is $96.78 \%$, $98.66 \%$, and $96.46 \%$ respectively.[4] Ozturk et al. proposed the DarkCovidNet Model for the identification of COVID-19 from chest X-ray images.[6] They evaluate their model on a datasets including 127 chest $X$-ray images of COVID-19 infection as well as 500 healthy and 500 pneumonia chest X-ray images.[6] Their model produced a classification accuracy of $98.08 \%$ for binary classes (COVID-19 vs. Healthy) and $87.02 \%$ for multiclass cases (COVID-19 vs. healthy vs. pneumonia).[6]

In this study we use Deep Transfer Leaning to iden- 
Table 1. Number of X-ray images in each class split.

\begin{tabular}{lccc}
\hline Split & COVID-19 & Healthy & Pneumonia \\
\hline Training set & 247 & 799 & 1598 \\
Testing set & 62 & 201 & 402 \\
\hline
\end{tabular}

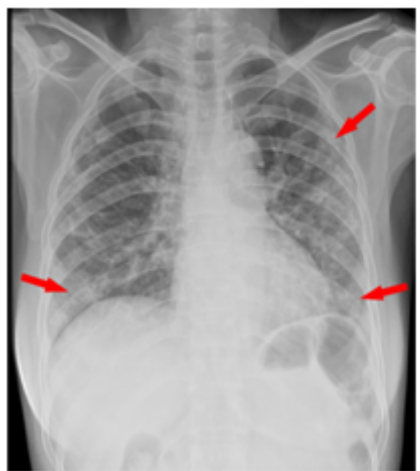

COVID-19

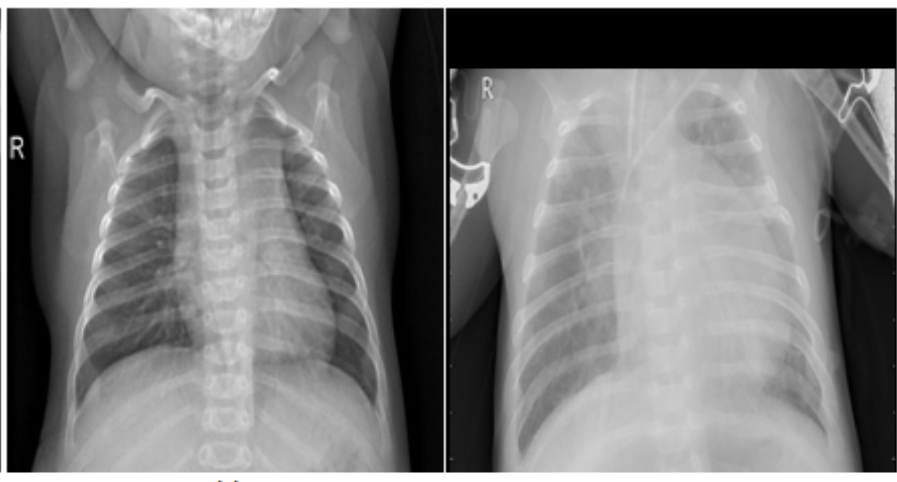

Healthy

Pneumonia

Figure 1. An example image from each class in the dataset

tify COVID-19 infection on a relatively large COVID-19 dataset (without augmentation) comparing with previous studies, in order to get more reliable diagnostic performance, based on two evaluation metrics; sensitivity and specificity.

\section{Methods}

\section{Datasets}

A total of 309 COVID-19 chest X-ray images (excluding lateral images) are collected; 236 COVID-19 images are obtained from the datasets of Cohen et al. $[7,8]$ and 73 other COVID-19 images are obtained from Kaggle dataset.[9] We also prepare 2,000 pneumonia and 1,000 healthy chest $\mathrm{X}$-ray images, collected from the dataset of Kermany et al.[10] All images are resized to $224 \times 224$ pixels. We split our datasets into training set $(80 \%)$ and testing set $(20 \%)$, as it is descript in Table 1 above. Data augmentation techniques are not used in this study.

\section{Models}

The Deep Transfer Learning method is used in this study, because the samples in our datasets are small and not sufficient to train a CNN model from onset.[2,4,5] Transfer learning consists of extracting features learned on one problem, and using them on a new, similar problem.[11] In our case, we extract features learned on the ImageNet dataset, a large dataset including of $1.4 \mathrm{M}$ images and 1000 classes [5], and leveraging them in the detection of COVID-19 infection.
We fine-tune three pre-trained deep CNNs models on the train dataset: DenseNet 121 [12], NASNetLarge, and NAS-NetMobile.[1] Firstly, we instantiate the proposed models pre-loaded with weights trained on ImageNet datasets.[5] We do not include the classification layers at the top, to make the models ideal for feature extraction.[5] Then, we construct a new classifier and add it on top of the base models. The new classifier consists of a pooling layer and a dense fully connected layer. Finally, we freeze the convolutional base before training our models.

The training is done for 10 epochs, with a batch size of 16. We use ADAM optimization with a learning rate of 0.001 . We train our models with categorical cross entropy. The implementation is conducted in Python 3 using Keras [11] and Tensorflow [5].

We use sensitivity and specificity metrics to evaluate our models' performance, since the whole dataset is imbalanced.[2] These evaluation metrics are defined as follows [2]:

$$
\begin{aligned}
& \text { Sensitivity }=\frac{\text { True positive (TP) }}{\text { Total positive COVID-19 images }} \\
& \text { Specificity }=\frac{\text { True negative (TN) }}{\text { Total negative COVID-19 images }}
\end{aligned}
$$


Table 2. Sensitivity and specificity rates of DenseNet 121, NASNetLarge, and NASNetMobile models.

\begin{tabular}{lcc}
\hline Models & Sensitivity & Specificity \\
\hline DenseNet 121 & $98.40 \%$ & $99.80 \%$ \\
NASNetLarge & $100 \%$ & $99.50 \%$ \\
NASNetMobile & $100 \%$ & $99.30 \%$ \\
\hline Average & $99.45 \%$ & $99.50 \%$ \\
\hline
\end{tabular}

Table 3. Confusion matrices of the proposed models.

\begin{tabular}{llccc}
\hline Models & Predicted & Healthy & COVID-19 & Pneumonia \\
\hline \multirow{4}{*}{ DenseNet 121 } & Healthy & 176 & 0 & 25 \\
& COVID-19 & 0 & 61 & 1 \\
& Pneumonia & 11 & 1 & 390 \\
& Healthy & 163 & 0 & 38 \\
NASNetLarge & COVID-19 & 0 & 62 & 0 \\
& Pneumonia & 11 & 3 & 388 \\
& Healthy & 189 & 0 & 12 \\
NASNetMobile & COVID-19 & 0 & 61 & 0 \\
& Pneumonia & 11 & 4 & 381 \\
\hline
\end{tabular}

\section{Results}

Table 2 shows the achieved sensitivity and specificity rates by the proposed models. NASNetLarge and NASNetMobile reach a sensitivity rate of $100 \%$, while DenseNet 121 outperforms the two other models in term of specificity rate.

Table 3 shows the confusion matrices of DenseNet 121, NASNetLarge and NASNetMobile models, respectively. We can see from this table that our models confuse in the identification between COVID-19 and pneumonia in some cases.

\section{Discussion}

In this study, we revisited the detection of COVID-19 form chest $X$-ray images using Deep Learning. In order to get a more reliable diagnostic performance, we used a relatively large COVID-19 dataset of real chest Xray images (without augmentation). Our datasets contains 309 chest $X$-ray images, 2000 pneumonia and 1000 healthy chest $X$-ray images. The datasets were split into a training set $(80 \%)$ and a test set $(20 \%)$. We fine-tuned three pre-trained deep CNNs mod- els: DenseNet 121, NASNetLarge, and NASNetMobile.

We evaluated the performance of our models on the test dataset based on two evaluation metrics: sensitivity, specificity. The proposed models show a good COVID19 diagnostic performance, where they achieve an average sensitivity rate of approximately $99.45 \%$ and an average specificity rate of approximately $99.5 \%$. From the confusion matrices, we see that these models confuse in the detection between COVID-19 and pneumonia in some cases.

Since the whole dataset is imbalanced, more metrics are needed to evaluate the performance of our models, such as, the ROC curves and Precision-Recall curves. The used set of COVID-19 X-ray images in this study is limited. Thereby, a larger COVID-19 image dataset could lead to a more reliable diagnosis.[2] The automatic diagnosis of COVID-19 using artificial intelligence could be helpful, but should not replace clinical diagnosis. 
Received: June 24, 2020

Accepted: June 26, 2020

Published: June 23, 2020

Copyright: (c) 2022 The author(s). This original article is brought to you for free and open access by ThinkIR: The University of Louisville's Institutional Repository. For more information, please contact thinkir@louisville.edu. This article is distributed under the terms of the Creative Commons Attribution 4.0 International License (CC BY 4.0), which permits unrestricted use, distribution, and reproduction in any medium, provided the original author and source are credited.

Funding Source: The author(s) received no specific funding for this work.

Conflict of Interest: All authors declared no conflict of interest in relation to the main objective of this work.

\section{References}

1. World Health Organization. Pneumonia of unknown cause - China. Available at: https://www.who.int/csr/don/05january-2020-pneumonia-of-unkown-cause-china/en/. Accessed 1 June 2020.

2. Minaee S, Kafieh R, Sonka M, Yazdani S, Soufi GJ. Deep-COVID: Predicting COVID-19 from chest X-ray images using deep transfer learning. arXiv [Preprint]. 2020 doi: http://arxiv.org/abs/2004.09363.

3. Boudrioua MS, Boudrioua A. Predicting the COVID-19 epidemic in Algeria using the SIR model. medRxiv [Preprint]. 2021 doi: 10.1101/2020.04.25.20079467.

4. Apostolopoulos ID, Mpesiana TA. COVID-19: Automatic detection from X-ray images utilizing transfer learning with convolutional neural networks. Phys Eng Sci Med 2020; 43(2):635-40. doi: 10.1007/s13246-020-00865-4. PMID: 32524445 .

5. TensorFlow. Transfer learning with a pretrained convnet. Available at: https://www.tensorflow.org/tutorials/ images/transfer_learning. Accessed 3 June 2020.

6. Ozturk T, Talo M, Yildirim EA, Baloglu UB, Yildirim $\mathrm{O}$, Rajendra Acharya U. Automated detection of COVID19 cases using deep neural networks with X-ray images. Comput Biol Med 2020; 121:103792. doi: 10.1016/j.compbiomed.2020.103792. PMID: 32568675.

7. Cohen JP, Morrison P, Dao L. COVID-19 image data collection. arXiv [Preprint]. 2020 doi: http://arxiv.org/abs/2003.11597.
8. Cohen JP, Morrison P, Dao L, Roth K, Duong TQ, Ghassemi M. COVID-19 image data collection: Prospective predictions are the future. arXiv [Preprint]. 2020 doi: http://arxiv.org/abs/2006.11988.

9. Dadario AMV. COVID-19 $X$ rays: $X$ rays and CT snapshorts of COVID-19 patients. Available at: https:// www.kaggle.com/andrewmvd/convid19-x-rays/metadata. Accessed 5 June 2020 .

10. Kermany DS, Goldbaum M, Cai W, et al. Identifying medical diagnoses and treatable diseases by imagebased deep learning. Cell 2018; 172(5):1122-31.e9. doi: 10.1016/j.cell.2018.02.010. PMID: 29474911.

11. Chollet F. Transfer learning \& fine-tuning. Available at: https://keras.io/guides/transfer_learning/. Accessed 2 June 2020.

12. Huang G, Liu Z, Maaten LVD, Weinberger KQ. Densely connected convolutional networks. In: 2017 IEEE Conference on Computer Vision and Pattern Recognition (CVPR), 2017:2261-9.

13. Zoph B, Vasudevan V, Shlens J, Le QV. Learning transferable architectures for scalable image recognition. In: 2018 IEEE/CVF Conference on Computer Vision and Pattern Recognition, 2018:8697-710. 